\title{
Fixing of volume holograms in ferroelectric $\mathrm{K}_{1-y} \mathrm{Li}_{y} \mathrm{Ta}_{1-x} \mathrm{Nb}_{x} \mathrm{O}_{3}$
}

\author{
Xiaolin Tong, Rudolf Hofmeister, Min Zhang, and Amnon Yariv \\ Department of Applied Physics, California Institute of Technology, Pasadena, California 91125 \\ Aharon Agranat
Department of Applied Physics, Hebrew University of Jerusalem, Jerusalem 91904, Israel
}

Victor Leyva

Ortel Corporation, Alhambra, California 91803

Received June 13, 1996

\begin{abstract}
We report on the fixing of photorefractive volume holograms in potassium lithium tantalate niobate with ionic gratings and also with ferroelectric domain-reversed gratings. A diffraction efficiency of $55 \%$ is obtained with domain reversal in a 2-mm-thick ferroelectric phase $\mathrm{K}_{1-y} \mathrm{Li}_{y} \mathrm{Ta}_{1-x} \mathrm{Nb}_{x} \mathrm{O}_{3}$ crystal doped with $\mathrm{Co}, \mathrm{V}$, and Ti. We measured the decay rate of the domain gratings and also of the initial electron gratings and ion gratings. The domain grating decay agrees with Vogel-Fulcher fits. The activation energies for ionic and electronic conductivity are 0.76 and $0.12 \mathrm{eV}$, respectively. ㄷ 1996 Optical Society of America
\end{abstract}

Volume holograms in photorefractive materials are formed by redistribution of charge carriers that are photoinduced by optical standing waves. These charges induce a space-charge field that causes a refractive-index grating that is due in the first order to the linear electro-optic effect. This grating can be converted into a fixed grating composed either of a mobile (at high temperature) nonphotoactive species, usually ions, ${ }^{1,2}$ or of reversed domains, ${ }^{3-6}$ which partially compensate for the electronic spacecharge field. Potassium lithium tantalate niobate $\left(\mathrm{K}_{1-y} \mathrm{Li}_{y} \mathrm{Ta}_{1-x} \mathrm{Nb}_{x} \mathrm{O}_{3} ; \mathrm{KLTN}\right)$ is a photorefractive crystal $^{-9}$ with a perovskite structure. The ferroelectric phase transition temperature is determined from 100 to $400 \mathrm{~K}$ by the $\mathrm{K} / \mathrm{Li}$ and $\mathrm{Ta} / \mathrm{Nb}$ ratios. The dominant charge carriers are either holes or electrons for 514.5-nm wavelength excitation and can be changed by oxidation/reduction treatment. In a previous Letter ${ }^{9}$ we investigated the fixing processes in KLTN crystals in the paraelectric phase. In what follows we present results of fixing photorefractive gratings in KLTN-doped crystals in the ferroelectric phase.

In our experiments we determined the ferroelectric phase transition temperature of the sample (KLTN:Co, $\mathrm{V}, \mathrm{Ti}$ ) by monitoring the temperature dependence of the low-frequency dielectric constant. The cubic-totetragonal transition occurred at $T_{c}=25^{\circ} \mathrm{C}$ for the sample used in the experiment. The sample was $7 \mathrm{~mm} \times 6 \mathrm{~mm} \times 2 \mathrm{~mm}$, cut along the crystallographic axes, and polished optically. $\mathrm{Ni}$ and Au were evaporated onto the $2 \mathrm{~mm} \times 6 \mathrm{~mm}$ face. We used a thermoelectric controller to provide a stable temperature mount enclosed in a vacuum chamber. We set the temperature to $35^{\circ} \mathrm{C}$ (in this case the sample is in the paraelectric phase) and cooled the sample to $5{ }^{\circ} \mathrm{C}$ (ferroelectric phase) at a rate of $1{ }^{\circ} \mathrm{C} / \mathrm{min}$. On cool- ing through the phase transition, the crystal became opalescent as light scattered from the domain walls. A field of $2000 \mathrm{~V} / \mathrm{cm}$ was then applied across the sample. The domains grew at $45^{\circ}$ to the direction of applied field. After several minutes the individual domains coalesced into a single domain. By integrating the current flow through the sample, we determined the spontaneous polarization of the sample to be $5 \mu \mathrm{C} / \mathrm{cm}^{2}$.

An Ar-ion laser beam at $\lambda=514.5 \mathrm{~nm}$ with a total intensity of $1 \mathrm{~W} / \mathrm{cm}^{2}$ was used to write the hologram (Fig. 1). The polarization of the writing beams was normal to the applied field to minimize beam-coupling effects, including beam fanning. The grating period was $1 \mu \mathrm{m}$. We calculated the diffraction efficiency as the ratio of the diffracted power to the power incident upon the crystal corrected for losses from facet reflections. We recorded holographic gratings at $70{ }^{\circ} \mathrm{C}$ for $20 \mathrm{~min}$. It was in the paraelectric phase where an applied field was used to utilize the quadratic electrooptical effect for monitoring the diffraction efficiency. The diffraction efficiency increased to a maximum in several minutes and then decreased to a lower level,

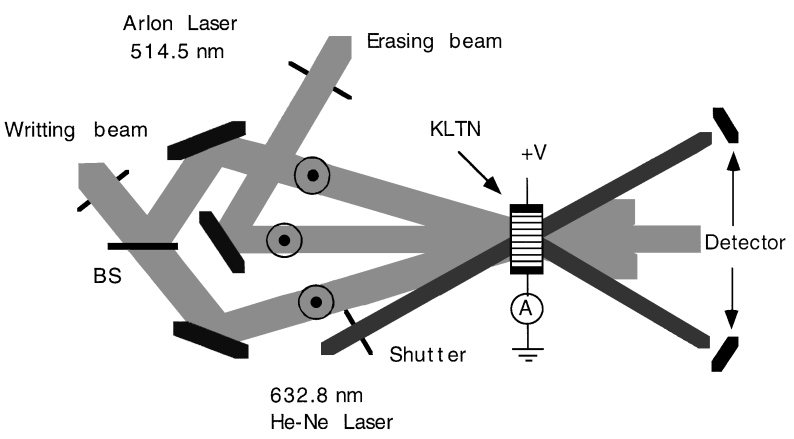

Fig. 1. Experimental setup for holographic writing, fixing, and readout. BS, beam splitter.

( 1996 Optical Society of America 
indicating that the ionic grating partially compensated for the electronic space-charge field. After recording the hologram that was compensated we erased the electronic contribution by illuminating the crystal with a non-Bragg-matched Ar-ion laser. We cooled the sample to different temperatures to measure the decay rate of the ion gratings. The fixed grating was read out by an extraordinarily polarized $\mathrm{He}-\mathrm{Ne}$ laser aligned at the Bragg angle.

The dielectric constant of the KLTN sample changes dramatically when the temperature is close to $T_{c}$. To avoid this influence we measured the diffraction when the dielectric constant of the sample was stable. The decay of the ionic gratings obeyed the Arrhenius form

$$
\tau_{T}=\tau_{0} \exp \left(E_{a} / K_{B} T\right) .
$$

An activation energy of $E_{a}=0.76 \mathrm{eV}$ and a constant $\tau_{0}=5 \times 10^{-9} \mathrm{~s}$ were fitted to the decay measurements (Fig. 2). The result differed slightly from the results with KLTN:Cu, V, Ti. ${ }^{9}$

The electronic grating was recorded at $-30{ }^{\circ} \mathrm{C}$. A weak beam $\left(0.1 \mathrm{~W} / \mathrm{cm}^{2}\right)$ and short writing times were used to prevent domain reversal. A Tektronix 2440 digital oscilloscope was used to monitor the displacement current flowing through the crystal. The writing period was $10 \mathrm{~s}$, and no external field was applied. Then we changed the temperature at each point to measure the decay of electron gratings. An external field of $1000 \mathrm{~V} / \mathrm{cm}$ was applied to enhance the diffraction efficiency while we measured the decay rate. When the temperature was lower than $10^{\circ} \mathrm{C}$ the ion mobility was negligible during measurement of the decay rate of the electron gratings. At this stage, if the electron gratings were erased with a non-Bragg-matched Arion laser beam, no other grating was revealed. This result indicated that it was the electron grating that decayed. Using Eq. (1), we determined an activation energy of $E_{a}=0.12 \mathrm{eV}$ and a time constant of $\tau=166 \mathrm{~s}$ (Fig. 3). This decay is due to the diffusion of shallow trapped electrons that compensated for the photorefractive space-charge field.

The domain grating was formed in the ferroelectric phase at $T=0{ }^{\circ} \mathrm{C}$. We used a monodomain sample for this experiment, with the poling procedure described above. The recording period was $30 \mathrm{~s}$. The displacement current across the sample was monitored (Fig. 1). The total beam intensity was $1 \mathrm{~W} / \mathrm{cm}^{2}$. We measured displacement current in two stages: (1) one incident beam and (2) two incident beams with the same total intensity. The Barkhausen current spikes were observed in stage 2 only (Fig. 2), which indicated that domain switching occurred because of the optically induced space-charge field. Exactly what configuration the domain walls took to compensate for the space-charge distribution is unknown. Both $90^{\circ}$ and $180^{\circ}$ domains exist in ferroelectric KLTN. We consider the $180^{\circ}$ domains the more likely candidate. We shone a $0.01-\mathrm{mW} / \mathrm{cm}^{2}$ $\mathrm{He}-\mathrm{Ne}$ beam to measure the diffraction from the domain gratings and used a $0.5-\mathrm{W} / \mathrm{cm}^{2}$ 514.5-nm beam to eliminate the electronic grating. The modulation of any self-enhancement ${ }^{10}$ was severely diminished with the 514.5-nm beam; thus the diffrac- tion came primarily from the domain grating. The diffraction efficiency of the domain gratings was measured at 55\%. The domain grating was erasable with a $3000-\mathrm{V} / \mathrm{cm}$ external field when $T>T_{c}-20^{\circ} \mathrm{C}$. If the temperature was lower than $T_{c}-30{ }^{\circ} \mathrm{C}$ the electric field would damage the sample before realigning the domains. If the beam intensity was greater than $\sim 4 \mathrm{~W} / \mathrm{cm}^{2}$ the thermoelectric cooler could not provide enough heat sink, and optical heating caused the sample local temperature to rise above $T_{c}$, so the crystal became paraelectric. Under these circumstances the domains would align randomly after the crystal was cooled to below $T_{c}$ in the absence of an external field. Domain grating decay is shown in Fig. 3. For

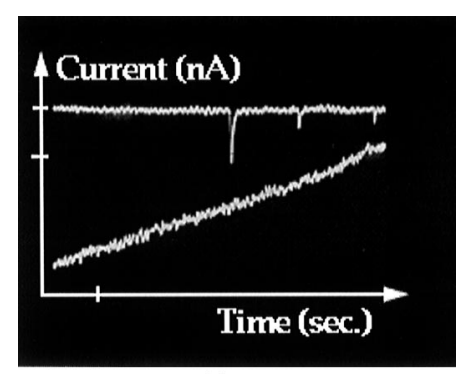

(a)

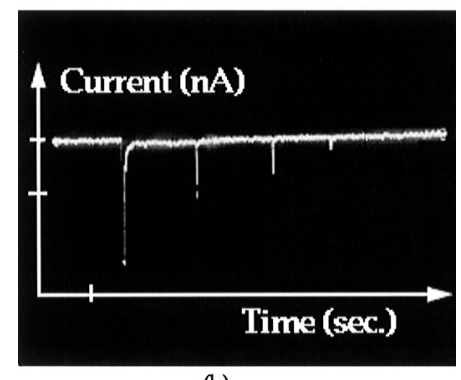

(b)

Fig. 2. Barkhausen jumps during recording of the hologram in a KLTN-doped crystal. (a) The total beam intensity $0.3 \mathrm{~W} / \mathrm{cm}^{2}$. The time scale is $1 \mathrm{~s}$. The upper curve represents current cross the crystal; the bottom curve is the diffraction efficiency. (b) The total beam intensity is $0.4 \mathrm{~W} / \mathrm{cm}^{2}$.

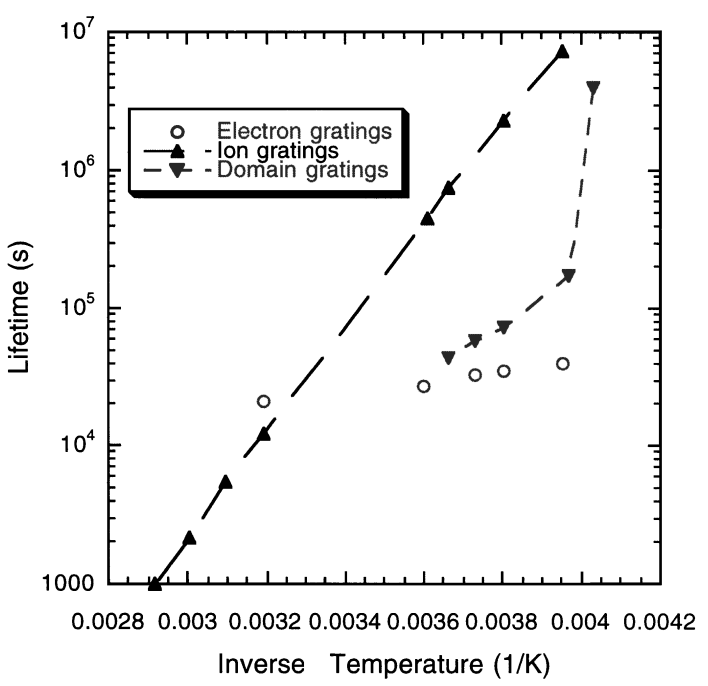

Fig. 3. Decay rate of ionic, electronic, and domain gratings in a KLTN:Co, Ti, V crystal. 
$T_{c}>T>-20^{\circ} \mathrm{C}$ the functional fit is an Arrhenius relation [Eq. (1)], with $E_{a}=0.27 \mathrm{eV}$ and a time constant $\tau=0.22 \mathrm{~s}$. For systems that have a polarizationfreezing temperature $T_{f}$ the Vogel-Fulcher law ${ }^{11}$ describes a freezing that occurs when the activation energy diverges. For Eq. (1)

$$
E_{a}=\frac{E^{\prime} T}{T-T_{f}},
$$

where $E^{\prime}$ is the depth of a potential well. The data from Fig. 3 indicate that $T_{f}=-35^{\circ} \mathrm{C}$; then $E^{\prime}=$ $13 \mathrm{meV}$ agrees well with the data. Apparently $T_{f}$ rises if $T_{c}$ is raised. This suggests that we can use high- $T_{c}$ crystals to store optical data in the ferroelectric phase by domain switching.

In summary, we have demonstrated domain switching that is due to the photorefractive space-charge field in ferroelectric phase KLTN:Co, V, Ti crystals. The diffraction efficiency of reversed domain gratings is $\sim 55 \%$ in a 2 -mm-thick crystal. There decay behavior for electron, ion, and domain gratings was characterized. For electron grating decay an activation energy $E_{a}=0.12 \mathrm{eV}$ and a time constant $\tau=166 \mathrm{~s}$ were obtained. For ion grating decay, $E_{a}=0.76$ and $\tau=5 \times 10^{-9}$. Domain grating decay obeys the VogelFulcher law, with a polarization-freezing temperature $T_{f}$ at $\sim T_{c}-60^{\circ} \mathrm{C}$. The lifetime of the domain grating is short unless the temperature is close to or lower than the polarization-freezing temperature.

The authors acknowledge the support of the Advanced Research Projects Agency University Research Initiative under U.S. Office of Naval Research grant N00014-920J-1891.

\section{References}

1. J. J. Amodei and D. L. Staebler, Appl. Phys. Lett. 18, 540 (1971).

2. L. J. Arizmendi, J. Appl. Phys. 65, 423 (1989).

3. F. Micheron and G. Bismuth, Appl. Phys. Lett. 20, 79 (1972).

4. M. Horowitz, A. Bekker, and B. Fischer, Appl. Phys. Lett. 62, 2619 (1993).

5. F. Kahmann, R. Pankrath, and R. A. Rupp, Opt. Commun. 107, 6 (1994).

6. A. S. Kewitsch, M. Segev, A. Yariv, and R. R. Neurgaonkar, Opt. Lett. 18, 1262 (1993).

7. A. Agranat, R. Hofmeister, and A. Yariv, Opt. Lett. 17, 713 (1992).

8. R. Hofmeister, A. Yariv, S. Yagi, and A. Agranat, Phys. Rev. Lett. 69, 1459 (1992).

9. V. Leyva, D. Engin, X. Tong, A. Yariv, and A. Agranat, Opt. Lett. 20, 1319 (1995).

10. M. Segev, A. S. Kewitsch, A. Yariv, and G. Rakujic, Appl. Phys. Lett. 62, 907 (1993).

11. G. J. Fulcher, J. Am. Ceram. Soc. 8, 339 (1925). 